touch sensations in exact mirror-symmetrical locations in the phantom. In two additional patients (R.L. and D.B.), such effects occurred only if the patient simultaneously 'saw' the phantom being touched - a curious form of 'synaesthesia' (14 out of 14 trials). Vibration was also referred intermanually in all four patients, but there was no referral of pain, heat or cold. For example, if patients J.P. or L.C. dipped their intact hand in a jar of cold water with ice cubes, they responded that they felt the 'cubes' in the phantom, but not the cold. These effects are not due to suggestion or confabulation for three reasons. First, touch and vibration were referred but not temperature or pain, and this was consistent across patients. Second, the patients experienced a latency of $2-4 \mathrm{~s}$ before the sensation was felt in the phantom. Third, the patients often expressed considerable surprise when they noticed these effects.

Even in normal individuals, the two hands may be linked in the brain as a result of frequent co-activation. Hence, sensory input from the left thumb might project not only to the right hemisphere but - by unidentified commissural pathways - to mirror-symmetrical points in the other hemisphere. This latent input may ordinarily be too weak to express itself, but when the right hand is amputated this input may become either disinhibited or progressively strengthened, so that touching the left hand evokes sensations in the right hand as well. The reason pain and temperature are not referred may be that there are no commissural pathways concerned with these modalities. In R.L. and D.B., however, the reactivation may not reach threshold amounts unless visual 'confirmation' is also provided (cells with bimodal receptive fields ${ }^{7}$ may be involved).

Our technique lends itself readily to brain-imaging studies. There must be considerable latent plasticity in the adult human brain. Precisely organized new pathways spanning the hemispheres can emerge in three weeks or less. Also, there must be great interaction between vision and touch, and so the strictly modular, hierarchical model of the brain currently in vogue must be replaced with a dynamic, interactive model in which 're-entrant' signalling ${ }^{8}$ plays an important role.

\section{S. Ramachandran}

\section{Rogers-Ramachandran}

\section{S. Cobb}

Brain \& Perception Laboratory, 0109,

University of California, San Diego,

La Jolla, California 92093-0109, USA

1. Melzack, R. Scient. Am. 266, 90-96 (1992).

2. Mitchell, S. W. Lippincotts Magazine for Popular Literature \& Science 8, 563-569 (1871).

3. Ramachandran, V. S. Cognitive Neurosci. Soc. Abstr. San Francisco (1995).

4. Ramachandran, V. S. Science 258, 1159-1160 (1992)

5. Ramachandran, V. S. Proc. natn. Acad. Sci. U.S.A. 90. 10413-10420 (1993).

6. Yang, T. T. et al. Nature 368, 592-593 (1994)

7. Gross, C. \& Graziano, M. Neuroscientist 1, 43-51 (1995).

8. Edelman, G. The Remembered Present (Basic Books, New York, 1989)

\title{
tRNA editing in metazoans
}

SIR - RNA editing, the modification of a transcript such that it differs from that inferred from its genomic sequence, is a common phenomenon in organelles of plants and unicellular organisms'. In metazoan mitochondria, polyadenylation is used to create termination codons ${ }^{2}$ and may be involved in the editing of several

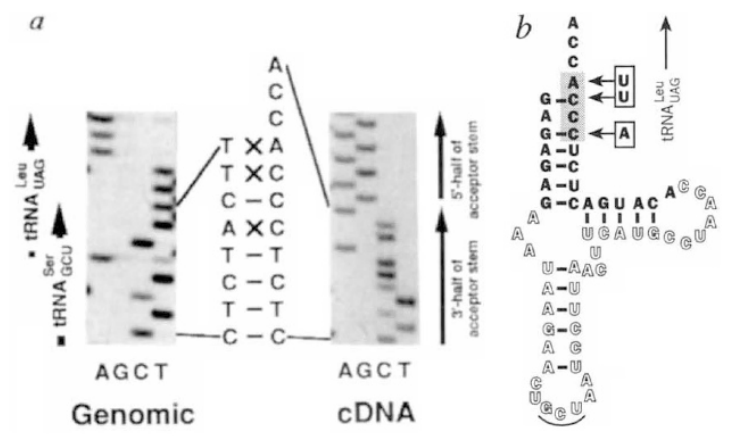

a, cDNA and gene sequences of the platypus mitochondrial seryl-tRNA anticodon GCU ( $\operatorname{tRNA}_{\mathrm{GCu}}^{\mathrm{Ser}}$ ). Only relevant parts of the autoradiographs of sequence ladders are shown. Differences between the genomic and CDNA sequences are indicated by crosses, identities by dashes. $b$, Inferred secondary structure of platypus mitochondrial tRNA $\mathrm{GCr}_{\mathrm{Ged}}^{\mathrm{Ser}}$. The region used for primers in CDNA synthesis and polymerase chain reaction $(\mathrm{PCR})$ are indicated by outlined characters. The region that in the gene overlaps with the downstream tRNA for leucine is indicated by the shaded background and the genomic versions of the edited nucleotide positions are boxed. tRNA circularization, cDNA synthesis, PCR and sequencing were carried out as in ref. 3. RNA of platypus and the clone carrying the platypus tRNA $A_{G C u}^{\text {Ser }}$ gene were gifts from $A$. Janke. The primer used for cDNA synthesis was 5'-GGATTAGCAGTTCTTATT-3' (platypus-S-FW). For amplification, this primer and 5'-TTAACTTCATGCCTAACC-3' (platypus-S-RV) were used.

transfer RNAs in land snails ${ }^{3}$. In addition, another RNA-editing mechanism changes an anticodon of a tRNA in marsupial mammals ${ }^{4,5}$. Here we show that the tRNA of a monotreme mammal is edited at three positions and that RNA editing is likely to be common in metazoan mitochondria.

The genomic sequence for the mitochondrial seryl-tRNA anticodon GCU of the platypus (Ornithorhynchus anatinus) ${ }^{6}, \mathrm{a}$ monotreme mammal, has two mismatches and one $\mathrm{G} \cdot \mathrm{U}$ base pair in its predicted aminoacylacceptor stem. In contrast, other features of the inferred tRNA structure are common to other seryl-tRNAs (data not shown). To investigate whether these unusual features are corrected by an RNAediting mechanism affecting the $5^{\prime}$ or $3^{\prime}$ halves of the acceptor stem, RNA was circularized $^{3}$ by T4 RNA ligase before synthesis of complementary DNA. The acceptor stem was then amplified, cloned and the nucleotide sequence of more than 20 clones determined.

It was found that one mismatched adenosine residue and the uridine residue of the G.U base pair in the $3^{\prime}$ part of the acceptor stem had been replaced by cytition, the discriminator base had changed adenine residue. Because the complete sequence of the mitochondrial genome of the platypus is known ${ }^{6}$ and import of cytoplasmic tRNAs into mitochondria is not believed to occur in vertebrates? ${ }^{7}$, the IRNA gene is unlikely to represent a pseudogene; more probably, an RNAediting mechanism changes the primary sequence of the tRNA post-transcriptionally.

RNA editing has recently been found to affect aminoacylacceptor stems of mitochondrial tRNAs in a protozoan (Acanthamoeba casellanii) ${ }^{8}$ and a land snail (Euhadra herklotsi) ${ }^{3}$. In the latter, the 13 changes observed in three tRNA species are similar to the three changes found in the platypus in that they involve events in the $3^{\prime}$ parts of the aminoacylacceptor stems. A further similarity between the platypus and land snail is that the genes of the tRNAs affected overlap with downstream genes encoded on the same strand. Such overlaps also exist in the mitochondrial genomes of various metazoans, for example, human, mouse and chiton ${ }^{9-11}$. Thus, the finding of tRNA editing in a land snail as well as the platypus makes it likely that an RNA-editing activity is involved in the processing of the transcripts in many, if not all, metazoan mitochondria.

\section{Shin-ichi Yokobori \\ Svante Pääbo}

Institute of Zoology,

University of Munich,

Luisenstrasse 14, PO Box 202126,

D-80021 Munich, Germany

1. Gray, M. W. Curr. Opin. Genet. Dev. 3, 884-890 (1993).

2. Cattaneo, R. A. Rev. Genet. 25, 71-88 (1991)

3. Yokobori, S. \& Pääbo, S. Proc. natn. Acad. Sci. U.S.A 92 (in the press)

4. Janke, A. \& Pääbo, S. Nucleic Acids Res. 21, 1523-1.525 (1993).

5. Mörl, M. Dörner, M. \& Pääbo, S. Nucleic Acids Res. 23 3380-3384 (1995)

6. Janke, A., Gemmell, N. J., Feldmaier-Fuchs, G., von Haeseler, A. \& Pääbo, S. J. molec. Evot. (in the press).

7. Roe, B. A., Wong, J. F. H., Chen, E. Y. \& Armstrong, P. W in Recombinant DNA Proc. 3rd Cleveland Symp. Macromolecules (ed. Walton, A. G.) 167-176 (Elsevier, Amsterdam, 1981).

8. Lonergan, K. M. \& Gray, M. W. Science $\mathbf{2 5 9}, 812-816$ (1993).

9. Anderson, S. et af. Nature 290, 457-465 (1981).

10. Bibb, M. J. et al. Cell 26, 167-180 (1981).

11. Boore, J. L \& Brown, W. M. Genetics 138, 423-443 (1994). 